\title{
Creating the Illusion of Motion in 2D Images
}

\author{
Reynold Bailey* \\ Washington University in St. Louis \\ Cindy Grimm ${ }^{\dagger}$ \\ Washington University in St. Louis
}
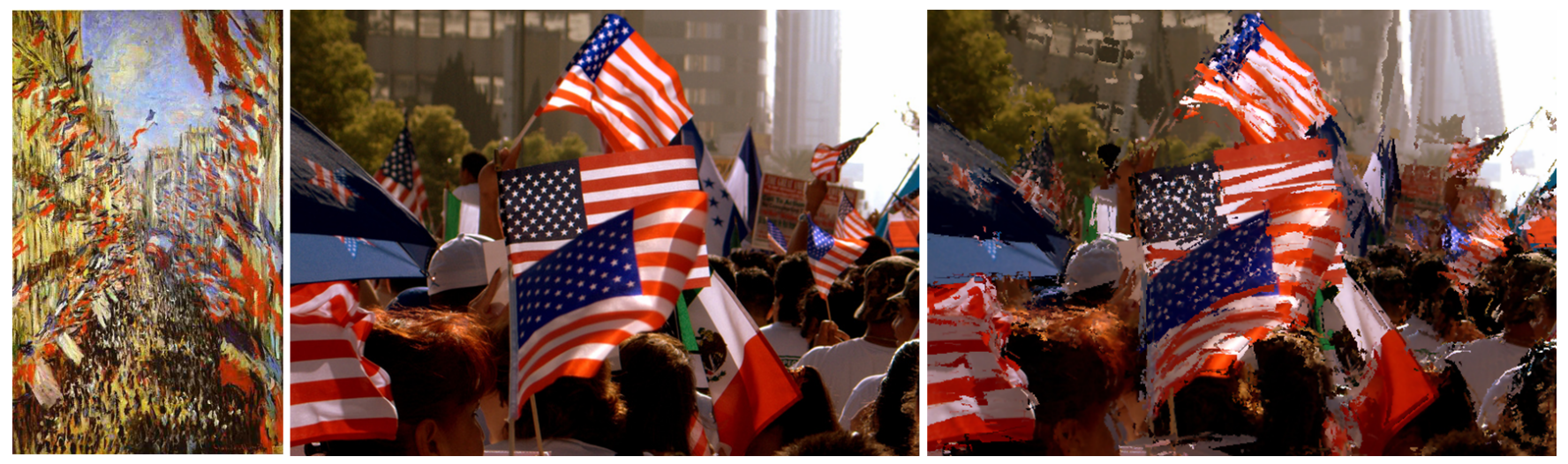

Figure 1: Left: Example of spatial imprecision to create the illusion of motion. Claude Monet, Rue Montorgueil - Paris, Festival of June 30, 1878 (1878). Center: Input image. Right: Output image.

\section{Introduction}

Traditional artists have developed several techniques for creating the illusion of motion in paintings. One common approach involves the use of spatial imprecision (misalignment of brush strokes) as illustrated in Figure 1 (left). When we first look at such paintings, our fast acting, low acuity peripheral vision gives us a rough idea of where the brush strokes are in the scene. Mentally, we join these brush strokes together to form a complete picture. This process is called illusory conjunction. It is only upon closer scrutiny with our slower, high acuity foveal vision that we notice that the strokes are misaligned. The illusion of motion is created because our visual system completes the picture differently with every glance (this explanation was adapted from Vision and Art: The Biology of Seeing [Livingstone 2002]).

We present preliminary results of a non-photorealistic technique that manipulates a static 2D image to produce the illusion of motion by introducing spatial imprecision (see Figure 1). Our technique consists of two steps. The input image is first segmented into regions of roughly uniform color and the resulting segments are then spatially perturbed. Our technique can be applied over the entire image or to specific regions of the image.

\section{Exposition}

Image segmentation can be performed using any existing approach. Each segment is assigned a unique ID and a local Cartesian co-

\footnotetext{
*e-mail:rjb1@cse.wustl.edu

†e-mail:cmg@cse.wustl.edu
}

ordinate system whose origin corresponds to the upper-left corner of the bounding box of the segment. Each segment undergoes a 2D spatial transformation (a rotation and a translation) with respect to its local coordinate system. The result shown in Figure 1 (right) was obtained by applying a random rotation (maximum 10 degrees) and a random translation (maximum 20 pixels) to each segment. Perturbing segments in this manner introduces holes in the image plane. We experimented with various inpainting approaches for filling these holes, but found that simply pasting the perturbed segments onto the original image gave better results.

\section{Conclusion and Future Work}

We present a novel approach for creating the illusion of motion in an image by introducing spatial imprecision. This work is still in its infancy and there are several issues that will be addressed as part of our future work. First, instead of naively performing random perturbations we plan to organize the perturbations to the individual segments to follow some structured spatial pattern. To some extent, this depends on the context of the image. The segments in an image depicting a windy scene, for instance, should in general be perturbed along the direction that the wind is blowing. From a userinterface standpoint, the simplest way to specify the desired spatial pattern is to allow the user to sketch a rough flow field directly into the image plane. Another issue that arises when perturbing segments (especially with random perturbations) is that perceptually relevant segments (such as faces) may get (partially) covered by other segments. To eliminate/minimize this problem, we plan to extend our framework to allow users to specify the relative importance of the various segments. The most important segments will then be processed last.

\section{References}

Livingstone, M. 2002. Vision and Art: The Biology of Seeing. Harry N. Abrams, Inc. 